\title{
Use of mesoporous silica as a reinforcing agent in rubber compounds
}

\author{
Betty L. López ${ }^{1}{ }^{*}$, León Dario Pérez ${ }^{1}$, Mónica Mesa ${ }^{1}$, Ligia Sierra ${ }^{1}$, Eric Devaux ${ }^{2}$,
} Mauricio Camargo $^{2}$, Christine Campagne ${ }^{2}$, Stéphane Giraud ${ }^{2}$

${ }^{1}$ Grupo de Ciencia de los Materiales, Instituto de Química, Universidad de Antioquia, A.A 1226, Medellín, Colombia; Fax 57-4-2330120; blopez@quimbaya.udea.edu.co

${ }^{2}$ Laboratoire de Génie et Matériaux Textiles (GEMTEX EA 2461), ENSAIT - Ecole Nationale Supérieure des Arts et Industries Textiles, 9 rue de l'Ermitage, BP 30329, 59056 Roubaix Cedex 01, France; Fax +33 (0)3 2027 25 97; eric.devaux@ensait.fr

(Received: November 9, 2004; published: March 19, 2005)

This work has been presented at the $12^{\text {th }}$ Annual POLYCHAR World Forum on Advanced Materials, January 6-9, 2004, in Guimaraes, Portugal

\begin{abstract}
Mesoporous silica is used as filler for styrene-butadiene rubber (SBR); filler-polymer interactions are compared with those exhibited when Ultrasil silica (VN3) is used. A silane coupling agent is added to improve filler dispersion and its influence on the bound-rubber formation is also investigated. The bound-rubber content is higher for the mesoporous silica and increases further for the sample containing silane. The increase is explained by chemical interactions between filler and rubber and penetration of the rubber chains into the mesopores. This is confirmed by ${ }^{13} \mathrm{C}$ solid-state NMR, IR spectroscopy and differential scanning calorimetry. Dynamic mechanical thermal analysis shows higher storage modulus for the rubber filled with mesoporous silica.
\end{abstract}

\section{Introduction}

Silica has been used as an important reinforcing agent in rubber compounds together with carbon black [1-5]. The mixing of silica into a rubber compound offers a number of advantages in tear strength, abrasion, heat resistance, hardness, high modulus, high resilience, improved rolling resistance, reduction in heat build-up, and increase in the compound adhesion of multicomponent products. However, silica does not provide the same level of reinforcement when compared to carbon black of the same particle size.

Reinforcement is usually defined as improvement in abrasion, tear, cutting, and rupture resistance, in stiffness and hardness of vulcanized compounds through the incorporation of finely divided (mineral) particles. The most important factor in the capability of certain minerals to impart reinforcement to elastomers is the average particle size. Reinforcement is readily obtained with particle sizes smaller than 100 $\mathrm{nm}$ and semi-reinforcement with particle sizes smaller than $1000 \mathrm{~nm}$. Particles larger than $10^{3} \mathrm{~nm}$ do not have reinforcing capabilities or have detrimental action [6], but 
the particles' chemical structure is also a decisive factor. Silica has a number of hydroxyl groups on its surface, which results in strong filler-filler interactions and adsorption of polar materials by hydrogen bonds. Since intermolecular hydrogen bonds between hydroxyl groups on the surface of silica are very strong, silica can aggregate tightly exhibiting a complex geometry, from elementary particles to aggregates, agglomerates and clusters with dimensions from 0.05 to $40 \mu \mathrm{m}$. This property can cause a poor dispersion of silica in a rubber compound [7], which together with its poor chemical affinity for a non-polar elastomer like styrene-butadiene (SBR) reduces the interactions.

In order to make the silica-silica agglomerates smaller and to enhance the compatibility between SBR rubber and silica, it is necessary to modify the surface of silica by the addition of a silane coupling agent that reacts with silanol on the surface of silica to form a siloxane bond. The silane molecules bonded to the silica surface provide a better interaction with the SBR rubber molecules because they have a higher chemical compatibility and the silylation also weakens the inter-particle interaction.

Since the total specific surface area of the particles and the pores of a filler give an important contribution to the filler's reinforcement properties, it is expected that the development of new mesoporous silicas with different properties, compared to precipitated silicas, such as high BET surface areas and their organized pore structures with pore sizes of $1.5-10 \mathrm{~nm}$, could increase the reinforcement because they present more sites to interact with the rubber matrix. Moreover, the rubber chains can penetrate the pores thus increasing the interaction. This effect of filler-rubber interactions is related to the occlusion or trapping of rubber in the pores or between or within aggregates, producing 'bound rubber'.

In order to have some understanding of the origin of the interactions, the present paper will be concerned primarily with the nature of mesoporous silica-styrenebutadiene rubber (SBR) interactions and the comparison with those exhibited for commercial precipitated Ultrasil (VN3). The effect on the elastic dynamic modulus is measured, since the desired properties for filled rubbers include good mechanical properties.

\section{Experimental part}

\section{Materials}

The mesoporous silicas were prepared from sodium silicate solution (commercial grade, 27 wt.- $\% \mathrm{SiO}_{2}, 11.9$ wt.- $\% \mathrm{Na}_{2} \mathrm{O}$ ). We used the non-ionic surfactant poly(ethylene glycol) 4-ter octylphenyl ether (Triton X100, from Aldrich), NaOH (Aldrich, analytical grade), and 5\% dimethyldichlorosilane in toluene (Supelco). The silica samples are known as Mesosil B.

Commercial SBR 1502 rubber from Monsanto was used (17 parts of styrene / 100 parts of butadiene; the latter was $85 \%$ butadiene $1-4$ and $15 \%$ butadiene $1-2$ ).

\section{Synthesis procedure of silica}

The samples were prepared according to the procedure reported before [8] with the following molar composition: $1 \mathrm{SiO}_{2}, n\left(1.20 \mathrm{Na}_{2} \mathrm{O}\right), 0.26$ Triton, $220 \mathrm{H}_{2} \mathrm{O}$. The amount of sodium was varied by adding $\mathrm{NaCl}$ in the synthesis, in order to modulate the polymerization rate and thus the silica's particle size. Mesosil-B10A and B10 samples 
were prepared by polymerization at $\mathrm{pH} 8.5$ and $n=1$ and $n=2$, respectively. Mesosil-B11A and B11 samples were prepared by polymerization at $\mathrm{pH} 6.5$ and $n=1$ and $n=2$, respectively.

The samples were calcined under air to remove the surfactant. The calcination consisted of heating at a rate of $1.5 \mathrm{~K} / \mathrm{min}$ up to $480^{\circ} \mathrm{C}$ and holding at this temperature for $3 \mathrm{~h}$.

\section{Silylation}

Silica samples were activated in vacuum at $200^{\circ} \mathrm{C}$. The samples were mixed with $5 \%$ dimethyldichlorosilane in toluene at $65^{\circ} \mathrm{C}$ during $3 \mathrm{~h}$ and finally washed several times with toluene and then with methanol to extract the excess of silane reagent and toluene. The samples were dried at $80^{\circ} \mathrm{C}$ for $4 \mathrm{~h}$. The amount of dimethyldichlorosilane needed for the reaction was calculated assuming that there are three silanol groups $(\mathrm{SiOH})$ per $\mathrm{nm}^{2}$, hence

$$
M_{\text {DMDCS }}=\frac{1000 \mathrm{~m}^{2}}{1 \mathrm{~g} \mathrm{silica}} \times \frac{3 \mathrm{SiOH}}{1 \mathrm{~nm}^{2}} \times \frac{10^{18} \mathrm{~nm}^{2}}{1 \mathrm{~m}^{2}} \times \frac{1 \text { molecule of DMDCS }}{2 \mathrm{SiOH}} \times \frac{128.99 \mathrm{~g} \text { of DMDCS }}{6.023 \times 10^{23} \text { molecules of DMDCS }}
$$

\section{Incorporation of the mesoporous silicas into the SBR-type elastomer}

In order to analyze interactions between rubber and silica, different mixtures were prepared under vulcanization conditions without the additives normally used. This means that the SBR-rubber becomes physically crosslinked and not vulcanised.

Before the preparation of rubber/silica mixtures, the mesoporous silicas were slightly crushed and passed through a Taylor \# 400 sieve in order to eliminate agglomerates larger than $40 \mu \mathrm{m}$. The silica + SBR mixtures, silylated and non-silylated, were prepared with 60 parts of silica for 100 parts of rubber, without cure activators, antidegradants, or curatives. Mixing conditions and time were kept constant in order to have the same mechanical interactions. For preparing the mixtures, the rubber was loaded into a laboratory mill roll (Francis Chaw, series 4934-01) and premixed for 1 $\mathrm{min}$, then the filler was added during $5 \mathrm{~min}$ and finally processed in a hydraulic press at $150^{\circ} \mathrm{C}$ for $15 \mathrm{~min}$ applying 4 tons.

A mixture of SBR and silica Ultrasil-VN3, commonly used as a reinforcement load for rubber, was prepared in the same way and used as reference for comparison with the results obtained with the mesoporous silicas. Ultrasil-VN3 silica has a BET surface around $170 \mathrm{~m}^{2} / \mathrm{g}$.

\section{Determination of the 'bound rubber'}

The mixture samples were cut in small pieces and put over a stainless steel sieve (40 $\mu \mathrm{m}$ meshes), then extracted with toluene for 7 days and with acetone for 1 day and finally dried for 1 day at $80^{\circ} \mathrm{C}$. Contents of bound rubber were determined from the weights of the samples before and after the extraction.

\section{Characterization of the materials}

The mesoporous silicas were characterized before preparing the rubber + mesoporous silica mixtures by: 
Microscopy: All samples were examined with an optical microscope (Axioskop Zeiss) equipped with a camera (IVC 800 12S) and a scanning electron microscope (SEM, Philips XL30) in order to determine particle morphology, size and aggregation.

Nitrogen adsorption/desorption: The isotherms were measured with an ASAP 2010 apparatus (Micromeretics) on calcined samples after activation at $300^{\circ} \mathrm{C}$ in vacuum for $6 \mathrm{~h}$. The calculation of the specific surfaces (BET), porous volumes and pore size distribution (BJH method) were performed with the software of the apparatus.

NMR spectrometry: ${ }^{29} \mathrm{Si}$ MAS NMR spectra were recorded using a Win Bruker 300 spectrometer, MAS with decoupling at $56 \mathrm{kHz}$; the pulse length was $2 \mu \mathrm{s}$ corresponding to a flip angle of $45^{\circ}$; recycling time was $1 \mathrm{~s}$; the number of scans was 8152; spun frequency $7 \mathrm{kHz}$; chemical shift reference: tetramethylsiloxane (TMS).

X-ray diffraction: XRD patterns were recorded from 1 to $10^{\circ}(2 \theta)$ with a Philips PW1130 diffractometer (Cu- $\mathrm{K}_{\alpha}$ radiation).

The rubber + silica mixtures were characterized by:

Differential scanning calorimetry (DSC): The samples were heated from 25 to $200^{\circ} \mathrm{C}$, kept at $200^{\circ} \mathrm{C}$ for $5 \mathrm{~min}$, cooled to $-75^{\circ} \mathrm{C}$, and heated again from -75 to $150^{\circ} \mathrm{C}$. The last run was analyzed. A TA Instruments model 2920 was used equipped with a refrigerated cooling system, using a heating and cooling rate of $10^{\circ} \mathrm{C} / \mathrm{min}$, under nitrogen atmosphere at a flow of $40 \mathrm{ml} / \mathrm{min}$.

Dynamic mechanical thermal analysis (DMTA): Samples of $1.5 \mathrm{~cm}$ length, $0.7 \mathrm{~cm}$ wide and $0.7 \mathrm{~cm}$ thin were analyzed from -100 to $20^{\circ} \mathrm{C}$ in a Polymer Lab model MKIII, using a strain value of $\pm 32 \mu \mathrm{m}$ and a frequency of $5 \mathrm{~Hz}$. The tests were performed at least three times on five samples of each material to ensure the reproducibility of the results.

NMR spectrometry: ${ }^{13} \mathrm{C}$ CP/MAS NMR spectra were recorded with a Win Bruker spectrometer in order to determine the degree of interaction between silica and rubber: using: $\pi / 2$ pulses of $4.5 \mu \mathrm{s}$, a rotation frequency of $7.5 \mathrm{kHz}$ and a recycling time of $1 \mathrm{~s}[8,10]$.

Infrared spectrometry: The silica + rubber mixtures that were too opaque or too thick were analyzed by the attenuated total reflectance (ATR) FTIR technique. $\mathrm{KBr}$ pellets were prepared for the silicas and toluene-extracted rubber + silica samples and analyzed by transmittance with a Perkin Elmer spectrophotometer, model Spectrum One.

\section{Characterization of the mesoporous silicas}

In general, measuring the specific surface area has been a standard practice in studies of filled rubbers. Specific surface area information along with structure information about the filler can be combined to give an idea how a rubber will be reinforced. Much is still unknown concerning the mechanisms of how these properties are related to the reinforcement and what the exact mechanisms of interaction are.

Tab. 1 shows the results of the characterization of the mesoporous silicas by XRD, SEM and nitrogen adsorption/desorption. The sample B10A, synthesized at $\mathrm{pH} 8.5$ and low sodium content, has a large specific area and good porosity properties in the mesoporous range and small particle size since the polymerization at this $\mathrm{pH}$ is fast. These characteristics seem to be adequate to achieve good reinforcement. Sample B10 with high sodium content looks more agglomerated. At pH 6.5 the pore size is 
smaller; the samples look less homogenous morphologically. The pore size distribution obtained by nitrogen adsorption shows a significant microporous contribution. If the sodium content is high, the silica can precipitate without interacting with the surfactant giving both microporous and mesoporous material, especially at low synthesis $\mathrm{pH}$. The silicas without silylation show agglomeration, but after silylation the particles are dispersed, as shown in Fig. 1.

Tab. 1. Characteristics of mesoporous silicas ( $S$ specific surface, $V$ specific volume)

\begin{tabular}{|c|c|c|c|c|c|c|}
\hline \multirow[t]{2}{*}{ Sample } & \multirow[t]{2}{*}{ Synthesis $\mathrm{pH}$} & \multirow[t]{2}{*}{$\% \mathrm{Na}$} & \multicolumn{3}{|c|}{ Porosity properties } & \multirow[t]{2}{*}{ Morphology and particle size } \\
\hline & & & $\begin{array}{l}S \text { in } \\
\mathrm{m}^{2} / \mathrm{g}\end{array}$ & $\begin{array}{c}V \text { in } \\
\mathrm{cm}^{3} / \mathrm{g}\end{array}$ & $\begin{array}{l}\varnothing \text { in } \\
\mathrm{nm}\end{array}$ & \\
\hline VN3 & $>8.5-9.0$ & 7.0 & 170 & 0.20 & 1.6 & Particles $<1 \mu \mathrm{m}$ \\
\hline B10 & 8.5 & 23.2 & 575 & 0.69 & 3.5 & $\begin{array}{l}\text { Particles } 1 \text { - } 2 \mu \mathrm{m} \text { and some } \\
\text { agglomerates }\end{array}$ \\
\hline B10A & 8.5 & 7.8 & 629 & 0.59 & 3.2 & $\begin{array}{l}\text { Disperse isometric particles } \\
0.5-1 \mu \mathrm{m}\end{array}$ \\
\hline B11 & 6.5 & 26.7 & 575 & 0.34 & 2.4 & $\begin{array}{l}\text { Particles } 1 \text { - } 2 \mu \mathrm{m} \text { and some } \\
\text { agglomerates }\end{array}$ \\
\hline B11A & 6.5 & 9.36 & 946 & 0.52 & 2.6 & $\begin{array}{l}\text { Particles } 1 \text { - } 2 \mu \mathrm{m} \text { and some } \\
\text { agglomerates }\end{array}$ \\
\hline
\end{tabular}

A)

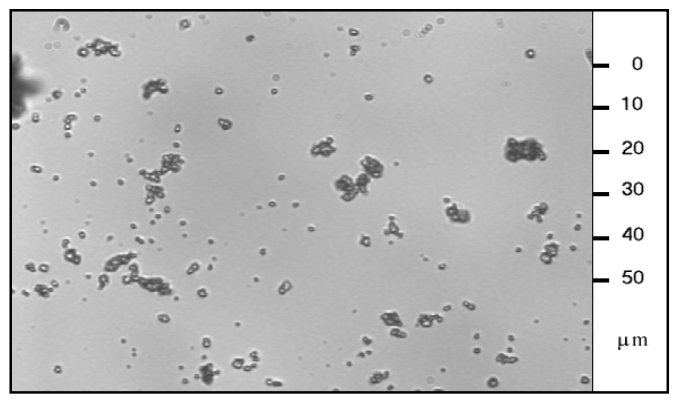

C)

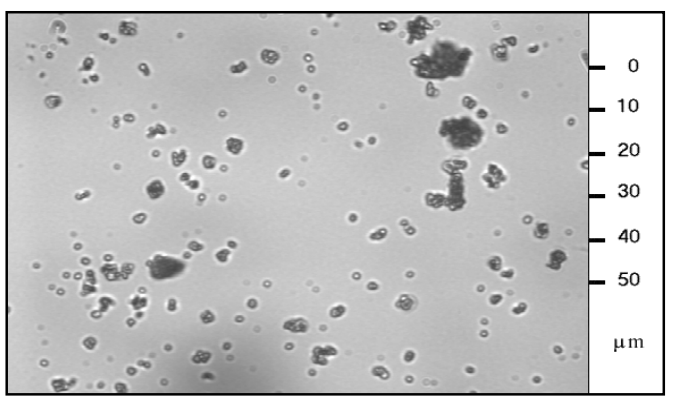

B)

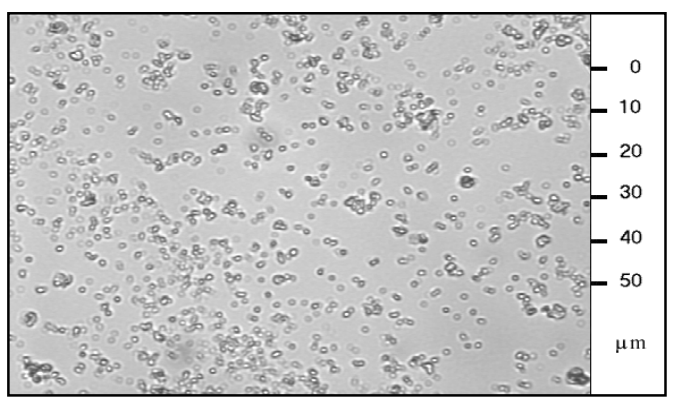

D)

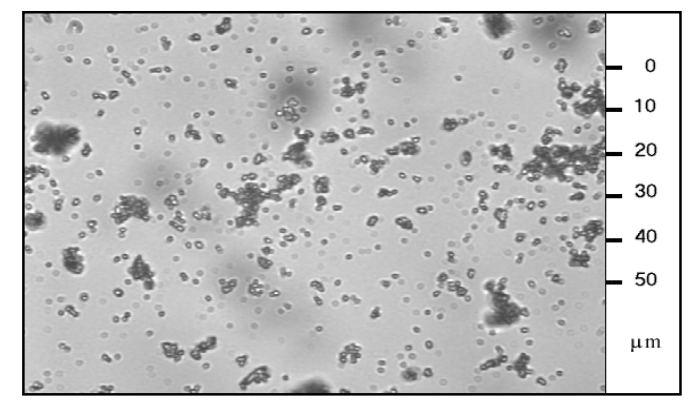

Fig. 1. Micrographs of silica samples: A) silica B10A without silylation, B) silica B10A silylated, C) silica B11A without silylation, D) silica B11A silylated

Silylated silicas were analyzed by $I R$ and ${ }^{29} \mathrm{Si}$ MAS NMR in order to determine the efficiency of the silylation reaction. Fig. 2 shows the ${ }^{29} \mathrm{Si}$ MAS NMR spectrum of VN3 using TMS as a chemical reference. It presents at $-6.2 \mathrm{ppm}$ the typical resonance of 
$\mathrm{SiO}\left(\mathrm{CH}_{3}\right)_{2} \mathrm{OH}$ and at $-13.9 \mathrm{ppm}$ that of $-\left(\mathrm{OSi}\left(\mathrm{CH}_{3}\right)_{2}-\mathrm{O}\right)_{-}$, thus indicating that the sample is silylated. There is also at $-100.7 \mathrm{ppm}$ a signal of $\mathrm{Si}(\mathrm{OSi})_{3} \mathrm{OH}$ and at 111.4 ppm a signal of $\mathrm{Si}(\mathrm{OSi})_{4}$.

FTIR transmittance spectra (not shown) of silylated silicas show the $\mathrm{C}-\mathrm{H}$ stretching and bending vibration bands of the dimethyl groups of the silane reagent that is attached to the silica.

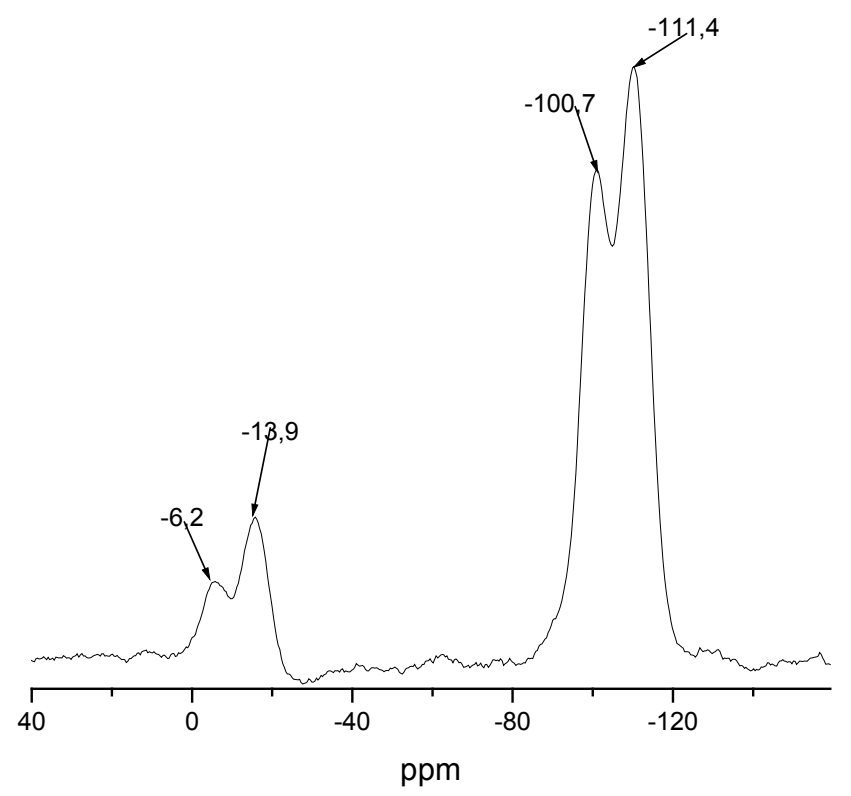

Fig. $2 .{ }^{29} \mathrm{Si}$ MAS NMR spectrum of silylated VN3 silica (VN3S)

\section{Characterization of the mixtures rubber + silica}

Determination of the bound-rubber content (BRC) in the mixtures

As already mentioned, bound rubber is the rubber that is trapped by the filler aggregates after mixing. The rubber chains are attracted either physically or chemically to form a rubber shell on the surface of the silica particles. The bound-rubber fraction of an uncured compound is the amount of rubber that is not extracted when it is exposed to a good solvent.

The BRC is calculated from the weight of the residue after extraction $(b)$, the weight of the initial mixture $(m)$, and the amount of silica present in the initial mixture $(a)$, determined by calcination at $600^{\circ} \mathrm{C}$. The BRC can be related to the amount of rubber or silica in the mixture rubber + silica before the extraction, namely:

$\% \mathrm{BRC}$ (vs. rubber) $=[(b-a) /(m-a)] \cdot 100$

$\% \mathrm{BRC}($ vs. silica) $=[(b-a) / a)] \cdot 100$

The temperature and the amount of silica were kept constant since the bound-rubber content can change with the extraction temperature [9], or with the concentration of silica [10], if the bound rubber is bounded physically. Results are shown in Tab. 2.

The SBR/silica mixture prepared with B10A presents higher BRC than the one prepared with Ultrasil-VN3, commonly used as a rubber reinforcing filler. The mixtures prepared with the mesoporous silica with higher degree of agglomeration present lower BRC. This indicates that the physical interaction (surface forces as van der 
Waals or hydrogen bonding) is weaker as the surface area of the filler decreases. It is also observed that BRC increases with increasing the BET surface area of the silica.

Tab. 2. Bound rubber content (BRC) in SBR + silica mixtures

\begin{tabular}{lll}
\hline Mixtures $T \approx 150^{\circ} \mathrm{C}$ & $\% B R C /$ rubber & $\% B R C /$ silica \\
\hline SBR/Ultrasil VN-3 & 33.16 & 66.41 \\
SBR/ B10 & 30.07 & 60.23 \\
SBR/ B10S ${ }^{\text {a) }}$ & 43.27 & 86.68 \\
SBR/B10A & 48.09 & 96.33 \\
SBR/B10AS $^{\text {a) }}$ & 78.12 & 156.47 \\
SBR/B11 $_{\text {SBR/B11S }}$ ) & 19.31 & 38.68 \\
SBR/B11A & 32.13 & 64.35 \\
SBR/B11AS & 30.54 & 61.17 \\
silica. & 56.13 & 112.43
\end{tabular}

The silylated samples present higher BRCs than non-silylated ones, indicating that the interaction could be also chemical since there is more affinity between polymer and silica. The silica itself is highly polar and does not interact well with a non-polar elastomer like styrene-butadiene (SBR) but does have good interaction after being rendered hydrophobic.

According to these results the sodium content affects the BCR since it changes the morphology and porosity characteristics of the materials but it does not have a positive effect on the interaction between rubber and silica. In order to investigate the interactions between rubber and mesoporous silica for other purposes, rubber + B10A with higher BRC and rubber + VN3 samples, silylated and non-silylated, were studied and the results are compared.

\section{DMTA results}

Generally, the main relaxation temperature of the rubber, $T_{\alpha}$, shifts to higher temperatures by physical mixing of the filler into the rubber matrix. However, the differences in $T_{\alpha}$ of the various samples are only about $3^{\circ} \mathrm{C}$. Probably with this technique it is only possible to detect the $T_{\alpha}$ of the non-interacting rubber in the mixtures.

As shown in Tab. 3 and Fig. 3, the elastic storage modulus $G^{\prime}$ is higher at low temperatures $\left(T=-100^{\circ} \mathrm{C}\right)$ and even at room temperature $\left(T=25^{\circ} \mathrm{C}\right)$ for the mixtures prepared with the mesoporous silica B10A that showed the higher BRC. The bound rubber affects the properties of the compounds. The mixtures of VN3 and B10 that have approximately the same BRC show almost the same $G^{\prime}$ [11]. The intensity of the tan $\delta$ peak becomes higher for the mesoporous silica compared to silica VN-3. This phenomenon seems to be related to an increase of reinforcement efficiency. One notices that the change $\Delta G^{\prime}$ between these two temperatures is higher for the mesoporous silica. This implies that chain entanglements and filler-filler interactions are stronger in this case. 
Tab. 3. DMTA results: $\tan \delta$ and storage modulus $G$ ' for SBR + silica mixtures

\begin{tabular}{lccccc}
\hline Mixture & $T_{\alpha}$ in ${ }^{\circ} \mathrm{C}$ & Max. $\tan \delta$ & \multicolumn{3}{l}{$\log G^{\prime}$} \\
at $-100^{\circ} \mathrm{C}$ & at $25^{\circ} \mathrm{C}$ & at $T_{\alpha}$ \\
\hline SBR-VN3 & -41.7 & 0.79 & 9.1 & 7.1 & 8.0 \\
SBR-VN3 S & -44.2 & 0.94 & 9.2 & 6.8 & 8.1 \\
SBR-B10 & -46.1 & 1.20 & 9.17 & 6.8 & 8.0 \\
SBR-B10S & -41.0 & 1.27 & 9.12 & 6.8 & 7.8 \\
SBR B10A & -44.5 & 1.40 & 10.2 & 7.6 & 8.7 \\
SBR B10A S & -44.9 & 1.30 & 9.3 & 6.8 & 7.8
\end{tabular}
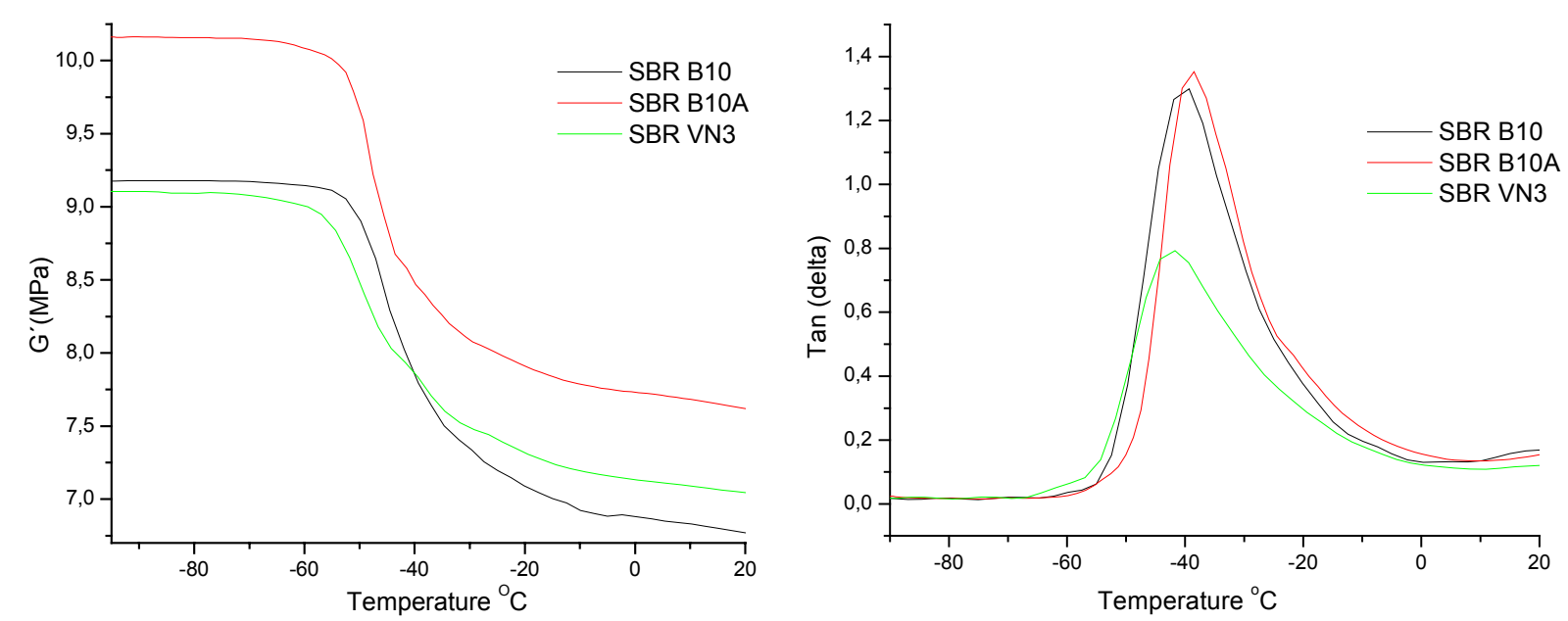

Fig. 3. DMTA results for the $S B R+B 10 A, S B R+B 10$ and $S B R+V N 3$ mixtures. Left: Storage modulus $G$ 'vs. temperature; right: $\tan \delta$ vs. temperature

Silylated silica rubber mixtures exhibit lower moduli compared with the non-silylated ones (Fig. 4); the silica-silica interaction, which is the primary mechanism of reinforcement especially at high filler loads, becomes weaker.
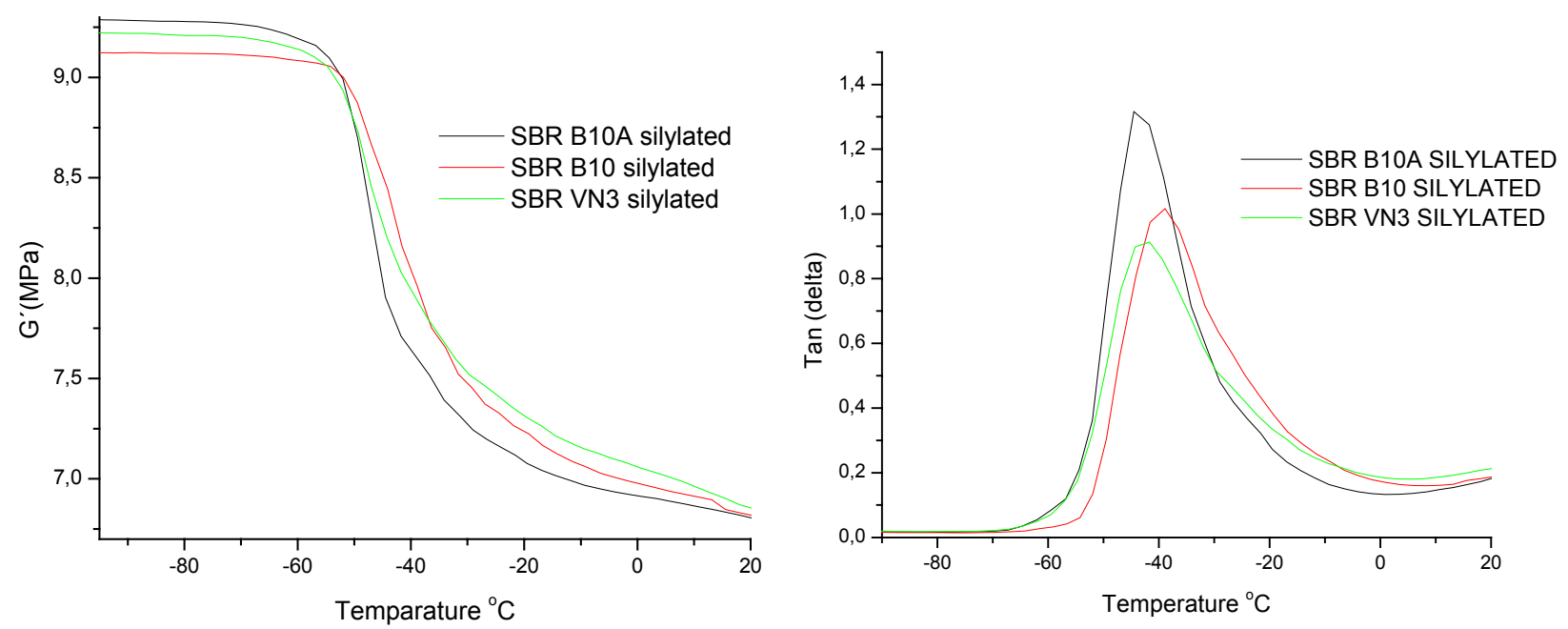

Fig. 4. DMTA results for SBR + silylated B10A, SBR + silylated B10 and SBR + silylated VN3. Left: Storage modulus $G$ 'vs. temperature; right: $\tan \delta$ vs. temperature 


\section{Infrared analysis}

Spectra of the silica + rubber mixtures are analyzed by attenuated total reflectance (ATR) FTIR. The spectra are shown in Fig. 5. The SBR spectrum shows C-H bending bands at $963 \mathrm{~cm}^{-1}$ of trans $1-4$ double bond units, at $909 \mathrm{~cm}^{-1}$ of 1,2 vinyl units (terminal alkene), at $759 \mathrm{~cm}^{-1}$ of $\mathrm{C}-\mathrm{H}$ wagging of cis 1,2 alkene, at $699 \mathrm{~cm}^{-1}$ of $\mathrm{CH}$ out-of-plane bending of polystyrene. We also see a band around $1703 \mathrm{~cm}^{-1}$ due to $\mathrm{C}=\mathrm{O}$ stretching more intense in the SBR + VN3 mixture, showing that the double bonds of the rubber get oxidized by air. The oxidation increases during milling and heating at $150^{\circ} \mathrm{C}$. The mixtures of mesoporous silicas do not show the $\mathrm{C}=\mathrm{O}$ band. This can be explained by the fact that the rubber chains can go inside of the mesopores; ATR is a superficial technique, and thus cannot detect them (Fig. 5a).

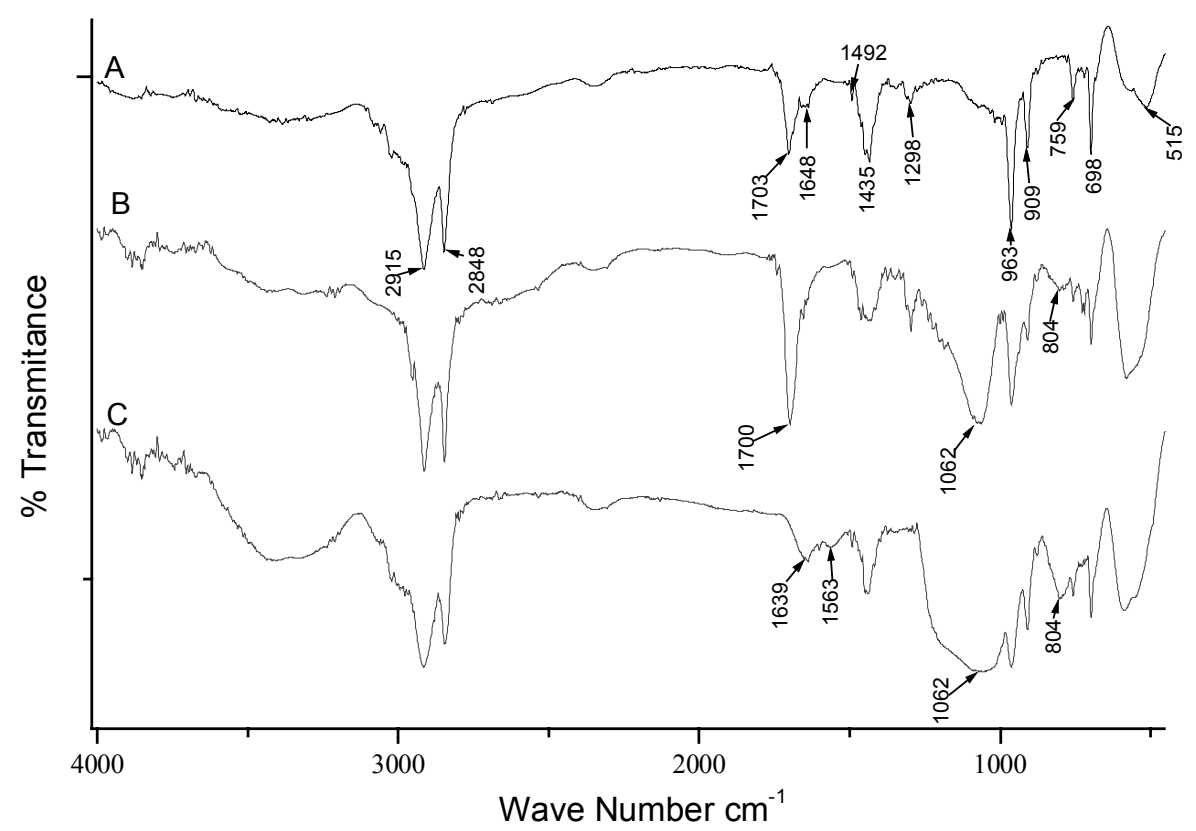

Fig. 5. Infrared spectra of A) non-filled SBR, B) SBR -VN3, C) SBR-B10A

The band at $1062 \mathrm{~cm}^{-1}$ due to $\mathrm{Si}-\mathrm{O}$ stretching vibration is broader for the mesoporous silica than for VN3 silica, and shows a shoulder around $1248-1160 \mathrm{~cm}^{-1}$. This could be due to $\mathrm{C}-\mathrm{O}$ stretching, suggesting the possibility of chemical interaction between rubber chains and silica. This possibility is higher for the mesoporous silica because some of the rubber chains can be inside of the mesopores. Formation of this group can result from free-radical reactions taking place during milling and heating of the silica + rubber mixtures. It is known that mesoporous silica can act as a free-radical deposit in reactions taking place during the thermal decomposition of polymeric materials by using them as catalysts [12].

The spectra of the mixtures prepared with silylated silica show an even broader band at $1062 \mathrm{~cm}^{-1}$ indicating higher interaction. When the mixtures are extracted with toluene, the transmission IR spectrum is made from $\mathrm{KBr}$ pellets. The presence of water in these samples is due to the hydrophilic character of the silica. The spectra show also differences in the absorption due to $\mathrm{SiO}_{2}$ stretching vibrations in the region $1250-1000 \mathrm{~cm}^{-1}$ (Fig. 6). Unfortunately SBR rubber exhibits also absorption in this region $(\mathrm{C}-\mathrm{H}$ bending of the trans $1-4$ double bond of butadiene and Si-O stretching vibration show a band at the same wave number of $963 \mathrm{~cm}^{-1}$ ) what makes difficult an 
interpretation of such differences in terms of the silica + rubber interactions. The spectra of extracted samples show a combination of the rubber + silica absorptions with the following features found for the mixture prepared with the mesoporous silica when compared with the VN3 silica sample: the $963 \mathrm{~cm}^{-1}$ band gets more intense, the shoulder around $1248-1160 \mathrm{~cm}^{-1}$ is well resolved, and the band of Si-O at 1100 $\mathrm{cm}^{-1}$ is shifted to lower wave number $\left(1087 \mathrm{~cm}^{-1}\right.$ for VN3 and $1060 \mathrm{~cm}^{-1}$ for B10A). This is due to the higher BRC, indicating that the interaction of the mesoporous silica with SBR is strong.

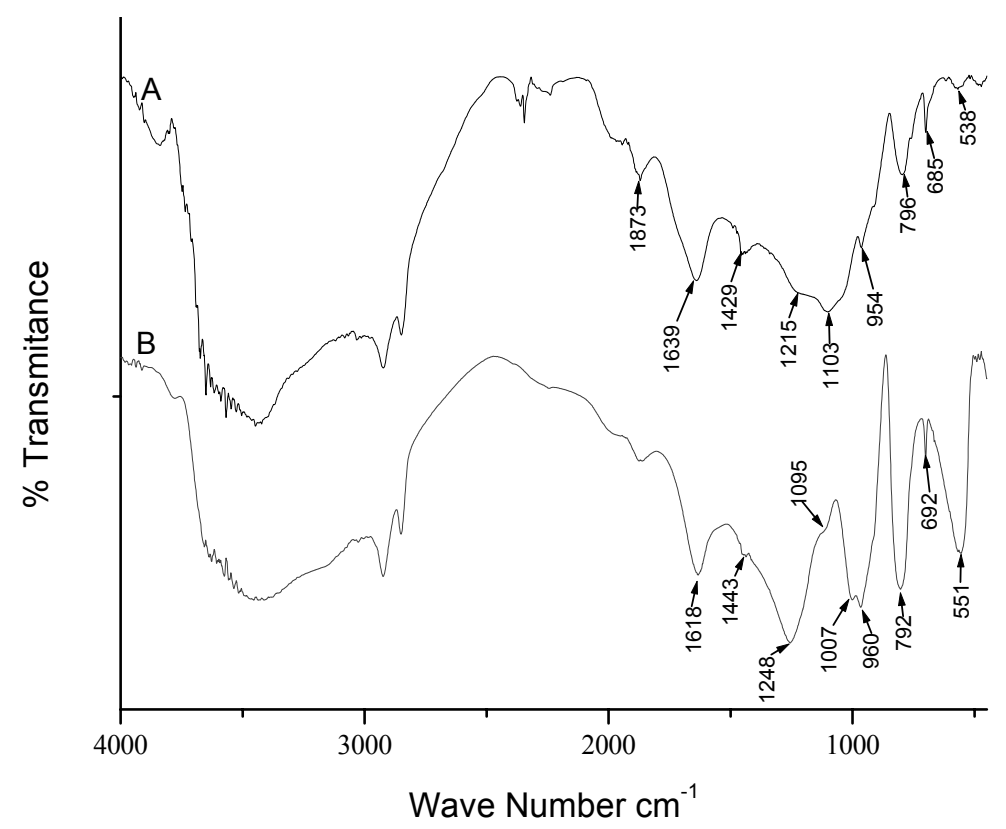

Fig. 6. IR spectra of A) SBR-VN3 extracted sample, B) SBR-B10A extracted sample

Kralevich et al. [13] concluded that the shifting and broadening of the absorptions in this region can be mainly attributed to physical interactions from electrostatic or dipole-induced forces but the presence of a new band at $1248 \mathrm{~cm}^{-1}$ could indicate chemical interactions.

\section{Differential scanning calorimetry}

DSC thermographs are shown in Fig. 7. The rubber sample and the non-extracted mixtures prepared with non-silylated and silylated VN3 silica present an endothermic peak at $31^{\circ} \mathrm{C}$ and at 21 and $28^{\circ} \mathrm{C}$, respectively. This is a melting peak since it is a reversible phenomenon that is still present after cooling and reheating. Since the peak is not present for the samples prepared with the mesoporous silicas, this could be another indication that the rubber chains are inside the mesopores and cannot reorganize themselves in order to crystallize.

$T_{\mathrm{g}}$ values of the SBR copolymer for the non-extracted samples change from $-55^{\circ} \mathrm{C}$ for the rubber alone to $-51^{\circ} \mathrm{C}$ for the $\mathrm{B} 10 \mathrm{~A}$ sample. These small changes are also observed by DMTA.

The transitions at $-5.35^{\circ} \mathrm{C}$, at -4.33 and at $-1.47^{\circ} \mathrm{C}$ can be attributed to rubber bonded to silica, which could present different interaction degrees: the rubber that is attached closest to the silica is much stiffer than the rubber molecules that are further away. 
These values are also affected by the rubber matrix itself because they change for the extracted samples.

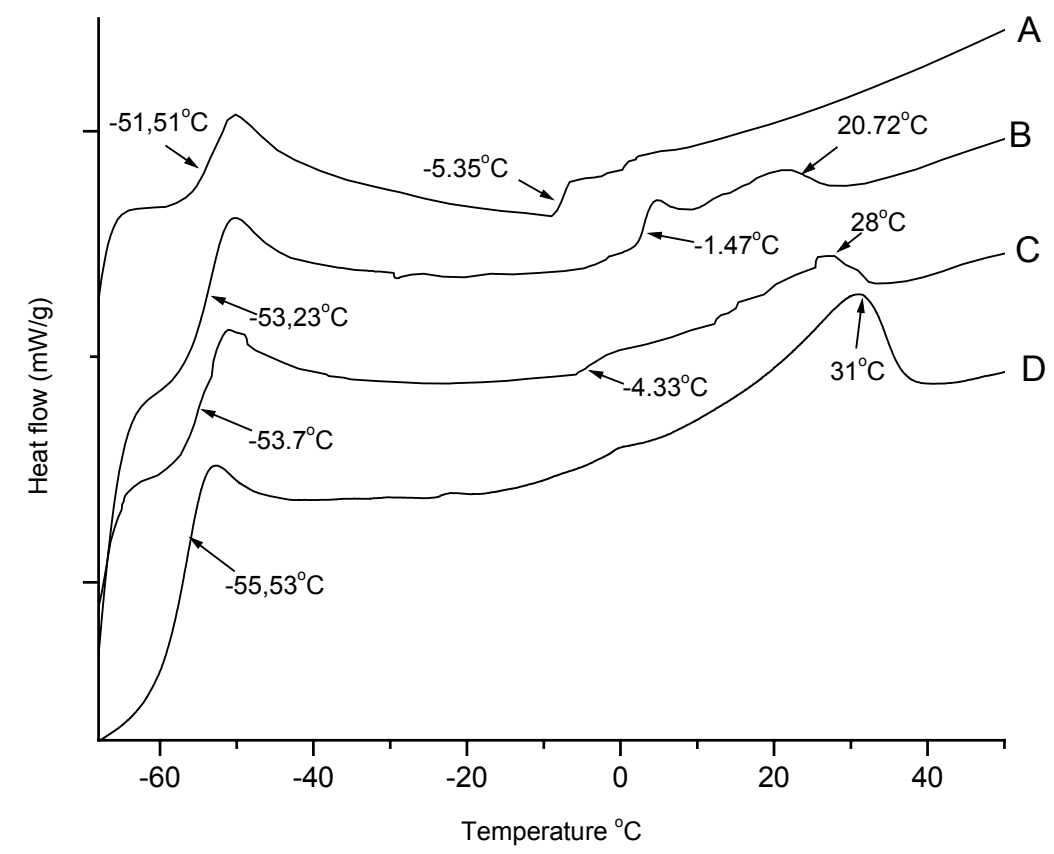

Fig. 7. DSC thermograms of non-extracted mixtures: A) SBR-B10A, B) SBR VN3 non-silylated, C) SBR VN3 silylated, D) non-filled SBR

In the non-extracted samples the $T_{\mathrm{g}}$ is $\approx-50^{\circ} \mathrm{C}$ corresponding to the unbound rubber that predominates in that case. In the thermograms of the extracted mixtures SBR + $\mathrm{B} 10 \mathrm{~A}$ and SNR $+\mathrm{VN} 3$, shown in Fig. $8, T_{\mathrm{g}}$ changes to $\approx-30^{\circ} \mathrm{C}$ for the bound rubber. The bound rubber consists of polymer chains with different kind of mobility, as those that are tightly bonded to the filler particles that are more constrained or chemically bound or those that are loosely bound or just dangling. This could be the reason for the presence of various transitions in the thermogram of the mixture prepared with B10A but not seen for the VN3 mixture.
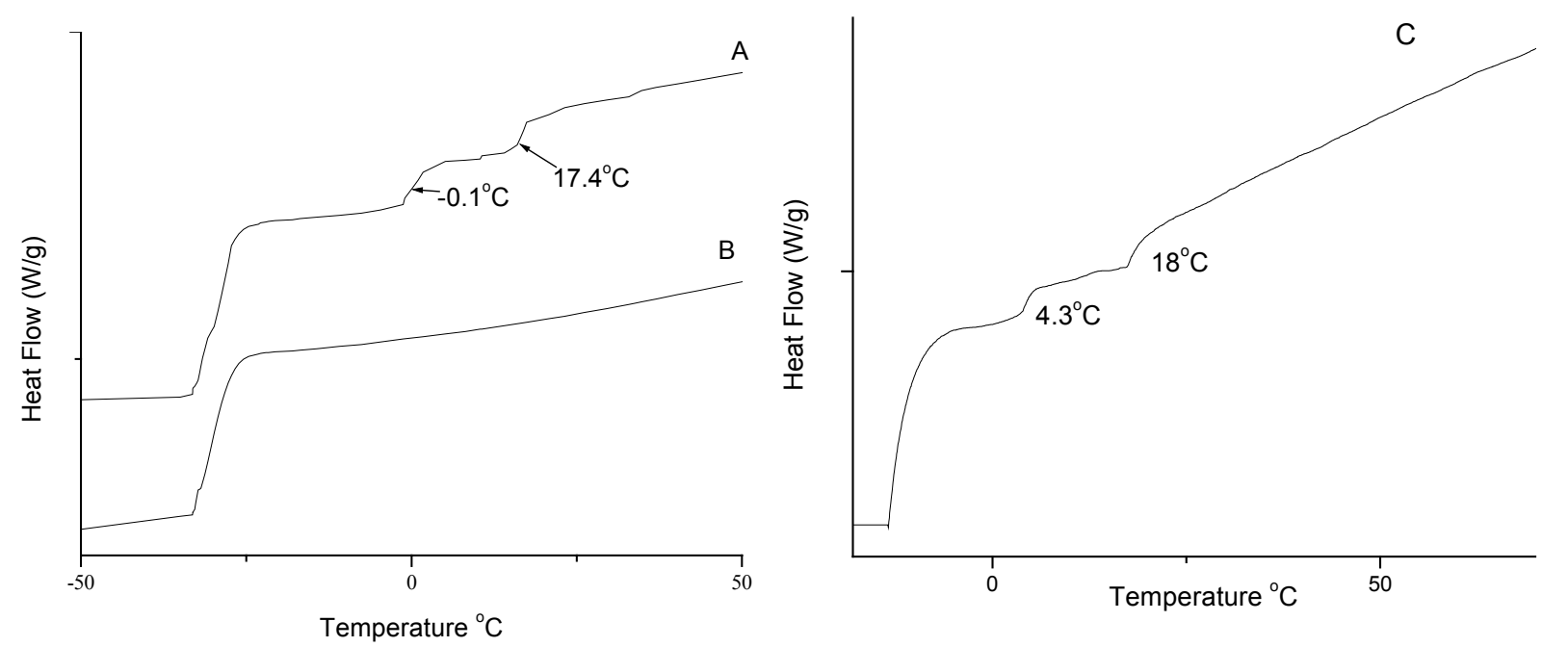

Fig. 8. DSC Thermograms of extracted SBR + silica mixtures: A) SBR-B10A, B) SBRVN3, C) SBR B10A silylated 


\section{8. ${ }^{13} \mathrm{C}$ CP/MAS NMR analysis of the extracted samples}

Fig. 9 shows the spectra of extracted samples. The characteristic peaks of SBR emulsion or solution appear at 27.6 for $\mathrm{C}$ of cis butadiene, 32.9 for $\mathrm{C}$ of trans butadiene, 114.5 for vinyl $\mathrm{CH}$ and 145.5 for the phenyl ring of styrene [14]. Because of the high glass transition temperature of SBR, spectra at room temperature exhibit broad bandwidths. Hence, it is not possible to distinguish the cis and trans carbon signals of butadiene, which are at $33.3 \mathrm{ppm}$; nor is it possible to distinguish the vinyl and phenyl carbons, which are at $130.3 \mathrm{ppm}$.

However, the SBR + B10A mixture shows also a different signal at $29.7 \mathrm{ppm}$; it can be assigned to aliphatic carbons in interaction with silica particles. This implies that the chemical environment of the butadiene chains interacting with mesoporous silica is different and we can observe them at different chemical shifts. This is another indication that the interaction is stronger for the rubber + mesoporous silica mixture than for the rubber + VN3 silica system.

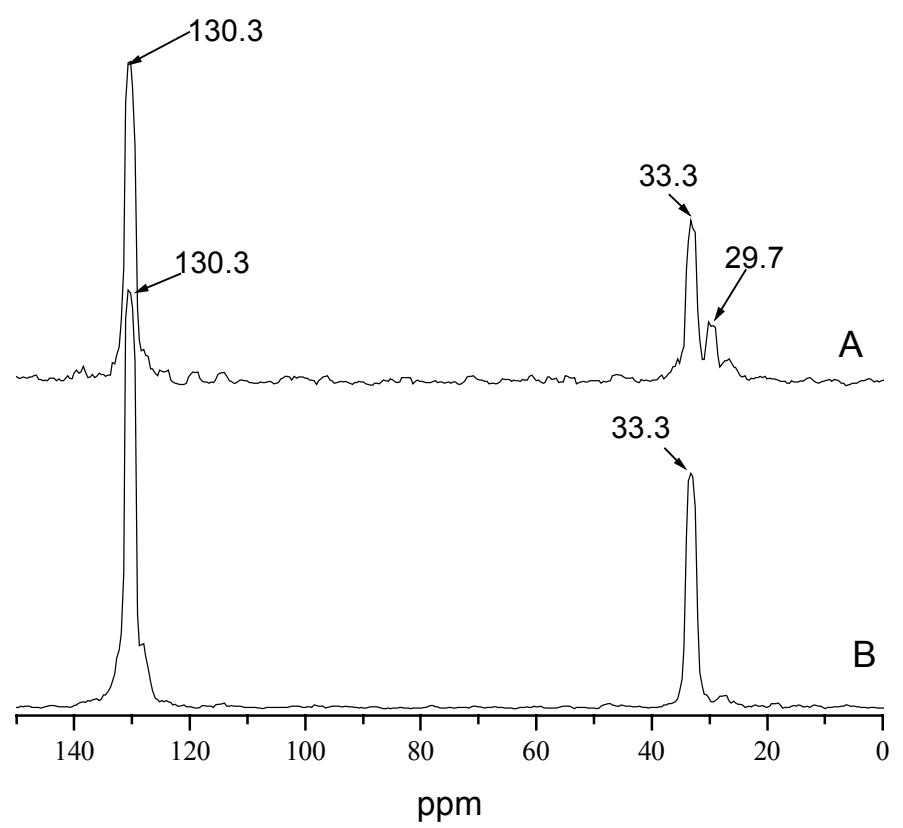

Fig. $9 .{ }^{13} \mathrm{C}$ CP/MAS NMR of extracted mixtures: A) SBR-B10A, B) SBR-VN3

Acknowledgement: NMR experiments were conducted in the Common Research Centre of the University of Lille. Mr. Bertrand Revel is acknowledged for helpful discussion and experimental assistance. We are grateful to the University of Antioquia, ENSAIT and ECOSNORD/COL-CIENCIAS/ICFES/ICETEX, both in Bogota, for financial support.

[1] Choi, S. K.; Polym. J. 1999, 7, 244.

[2] Byers, J. T.; Rubber World 1998, 218(6), 38.

[3] Wolff, S.; Wang, M. J.; Rubber Chem. Technol. 1992, 65, 329.

[4] Boonstra, B. B.; Cochrane, H.; Dannenberg, E. M.; Rubber Chem. Technol. 1975, 48, 558. 
[5] Voet, A.; Morawski, J. C.; Donnet, J. B.; Rubber Chem. Technol. 1977, 50, 342.

[6] Leblanc, J. L.; Prog. Polym. Sci. 2002, 27, 627.

[7] Li, Y.; Wang, M. J.; Zhang, T.; Zhang, F.; Fu, X.; Rubber Chem. Technol. 1994, 67, 693.

[8] Sierra, L.; López, B. L.; Guth, J. L.; Mater. Res. Innovat. 2002, 5, 268.

[9] Wolff, S.; Wang, M.; Tan, E.; Rubber Chem. Technol. 1993, 66, 163.

[10] Dutta, N.; Choudhury, N.; Haidar, B.; Vidal, A.; Donnet, J. B.; Delmotte, L.; Chezeau, J.; Polymer 1994, 35, 4293.

[11] Leblanc, J.; J. Appl. Polym. Sci. 1997, 66, 2257.

[12] Uddin, M. A.; Sakata, Y.; Muto, A.; Shiraga, Y.; Koizumi, K.; Kanada, Y.; Murata, K.; Microporous \& Mesoporous Mater. 1998, 21, 557.

[13] Kralevich, M. L.; Koening, J. L.; Rubber Chem. Technol. 1998, 71, 300.

[14] Pellicoli, L.; Mowdord, S. K.; Negroni, F.; Parker, D. D.; Koening, J. L.; Rubber Chem. Technol. 2001, 75, 65. 